\title{
Simplify your life: Towards more model-independent new physics searches
}

\author{
Matthew J. Dolan, ${ }^{a}$ JoAnne Hewett, ${ }^{b}$ Michael Krämer, ${ }^{c}$ and Thomas G. Rizzo ${ }^{b}$ \\ ${ }^{a}$ ARC Centre of Excellence for Particle Physics at the Terascale \\ School of Physics, University of Melbourne, 3010, Australia \\ ${ }^{b}$ SLAC National Accelerator Laboratory, Menlo Park 94025, CA, USA \\ ${ }^{c}$ Institute for Theoretical Particle Physics and Cosmology \\ RWTH Aachen University, 52056 Aachen, Germany \\ E-mail: dolan@unimelb.edu.au, hewetteslac.stanford.edu, \\ mkraemerephysik.rwth-aachen.de, rizzodslac.stanford.edu
}

\begin{abstract}
Simplified models provide a useful tool to conduct the search and exploration of physics beyond the Standard Model in a model-independent fashion. In this talk we consider the complementarity of indirect searches for new physics in Higgs couplings and distributions with direct searches for new particles, using a simplified model which includes a new singlet scalar resonance and vectorlike fermions that can mix with the Standard Model top-quark. We fit this model to the combined ATLAS and CMS $125 \mathrm{GeV}$ Higgs production and coupling measurements and other precision electroweak constraints, and explore in detail the effects of the new matter content upon Higgs production and kinematics. We highlight some novel features and decay modes of the top partner phenomenology, and discuss prospects for Run II.
\end{abstract}

XXIV International Workshop on Deep-Inelastic Scattering and Related Subjects

11-15 April, 2016

DESY Hamburg, Germany

\footnotetext{
* Speaker.
} 


\section{Introduction}

Two of the main objectives of the Large Hadron Collider (LHC) Run II are studying the detailed spectroscopy of the Higgs boson and continuing the search for physics Beyond the Standard Model (BSM). The properties of the recently discovered $125 \mathrm{GeV}$ Higgs boson provide a new tool in the search for new physics and may indirectly reveal its existence, whereas the gain in the center-of-mass energy of the collider in Run II supplies fertile ground to directly discover new particles.

So far, no indication for physics beyond the SM has been established, neither in the properties of the $125 \mathrm{GeV}$ Higgs boson, nor in direct searches for new particles. The Higgs boson properties can be characterised by the signal strength $\mu$, defined as the ratio of the measured Higgs boson rate to the prediction within the Standard Model. The ATLAS and CMS combination [1] of the signal strengths for various Higgs production and decay channels is shown in Fig. 1. The Higgs emerges from the data as expected within the Standard Model.
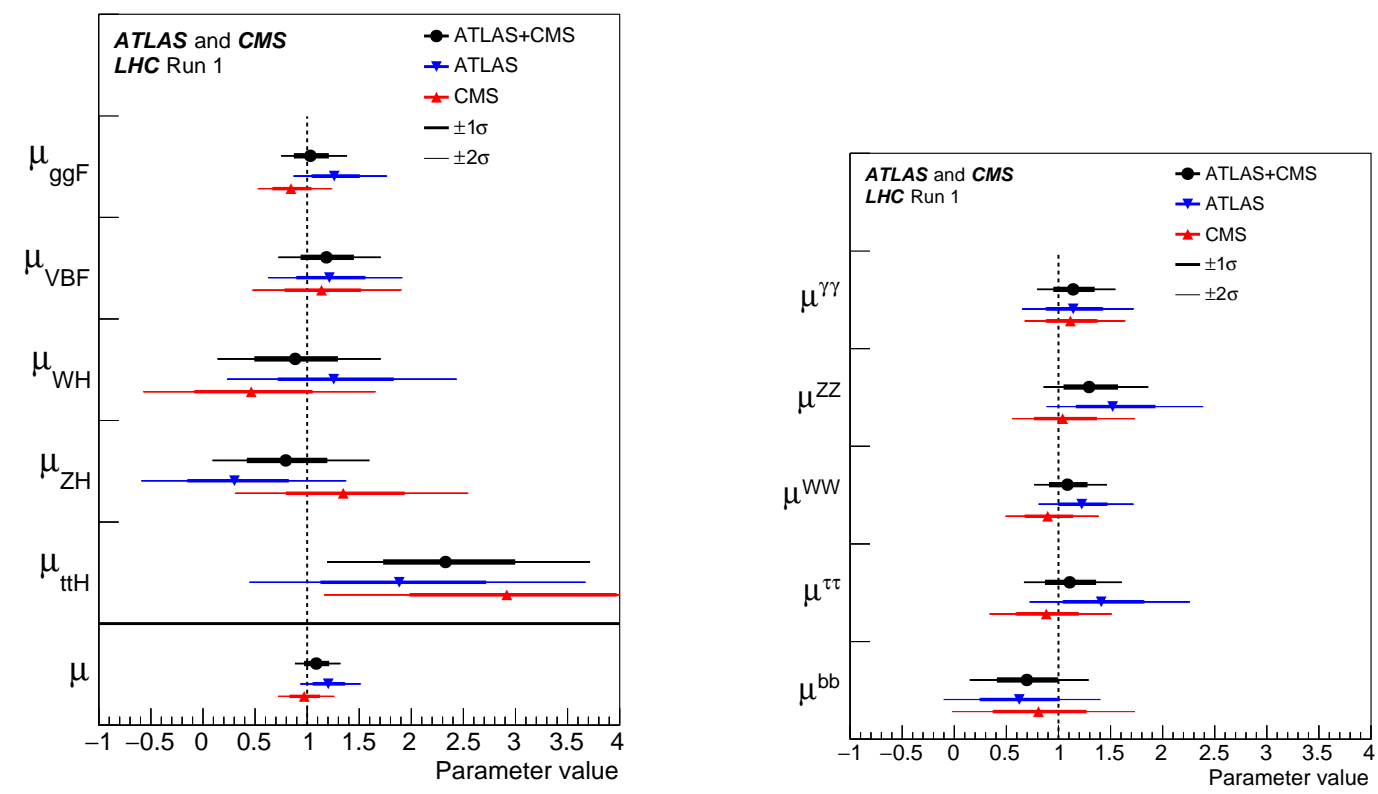

Figure 1: Best fit results for the Higgs production (left panel) and decay (right panel) signal strengths for the combination of ATLAS and CMS data. From Ref. [1].

Direct searches for new physics have not produced any deviation from the Standard Model expectation either, and limits have been placed on the masses and couplings of new heavy particles. The Higgs boson properties and the direct searches for new particles already probe energy and mass scales of $\mathscr{O}(\mathrm{TeV})$ and beyond. However, the searches are often theory-driven, and interpreted within specific classes of models. One may thus ask whether we actually search in all the right places, and whether the experimental results can be interpreted in a wider class of models.

A more generic and model independent approach to new physics searches relies on Simplified Models [2,3]. Simplified Models provide a framework for understanding the broad kinematic features of signatures using a small number of parameters, such as masses and couplings of new fields, without depending on specific characteristics of full UV-complete models. This focus on the 
relevant weak scale Lagrangian parameters allows for the design of relatively model-independent BSM searches that are broadly applicable to new physics scenarios.

The use of simplified models is not restricted to new physics searches, as they can also be of utility in understanding the limits set on new physics from precision Standard Model (SM) measurements such as the properties of the Higgs boson. Another approach often employed in this context is Effective Field Theory (EFT), where constraints are set on the Wilson coefficients of higher dimensional operators constructed out of Standard Model fields. A UV-complete theory can be mapped onto the EFT by integrating out the new heavy states, assuming there is a hierarchy of scales between the SM and new states. Simplified models differ in allowing the direct exploration of the phenomenology of the BSM states.

In this talk we consider a simplified model which includes a new singlet scalar resonance and vector-like fermions that can mix with the SM top-quark. We explore the complementarity of indirect searches for new physics in Higgs couplings and distributions with direct searches for new particles. The work presented in this talk has been published in Ref. [4], where also more details on the model and the phenomenology can be found.

\section{A Simplified Model}

In constructing a first simplified model for Higgs physics, we examine a scenario with minimal particle content and interactions that influences the $125 \mathrm{GeV}$ Higgs couplings in a calculable manner. We thus choose to add two ingredients to the SM: (i) a scalar singlet $S$, and (ii) a vector-like fermion $F$. The singlet $S$ acquires a vacuum expectation value (vev), $S=\left(s+v_{S}\right)$, and provides mass for the vector-like fermion, $m_{F}=y_{F} v_{S}$. The Higgs and new scalar fields mix via the term $\lambda_{H S} H^{\dagger} H S^{2}$, and thus generate new physics effects in both the gauge and fermionic SM Higgs couplings. Various choices for the quantum numbers of $F$ are possible, and different representations result in specific patterns for the Higgs cross sections and couplings. These are outlined in Table 1 for the Higgs gauge boson couplings $h V_{\mu \nu} V^{\mu \nu}$ induced by the vector-like fermion $F$. The gauge couplings are defined in terms of the coefficients $\varepsilon_{V}$ as

$$
\begin{array}{r}
\gamma \gamma: \varepsilon_{\gamma} \frac{\alpha}{\pi} \frac{1}{v_{H}}\left(\frac{\lambda_{H S} v_{H}^{2}}{m_{S}^{2}}\right), G_{a} G^{a}: \varepsilon_{g} \frac{\alpha_{S}}{\pi} \frac{1}{v_{H}}\left(\frac{\lambda_{H S} v_{H}^{2}}{m_{S}^{2}}\right), \\
B B: \varepsilon_{B} \frac{g^{\prime 2}}{\pi^{2}} \frac{1}{v_{H}}\left(\frac{\lambda_{H S} v_{H}^{2}}{m_{S}^{2}}\right), W_{i} W^{i}: \varepsilon_{W} \frac{g^{2}}{\pi^{2}} \frac{1}{v_{H}}\left(\frac{\lambda_{H S} v_{H}^{2}}{m_{S}^{2}}\right),
\end{array}
$$

and the $\varepsilon_{V}$ take on values as determined by the $F$ representation. Here, $v_{H}$ is the vacuum expectation value of the SM-like Higgs field $H$.

In this talk, for simplicity, we consider the case where $F$ is a color-triplet and $\mathrm{SU}(2)$ singlet vector-like fermion field, $T$, with charge $Q_{F}=+2 / 3$. We assume that the vector-like fermion mixes with the SM top-quark only. This model was chosen such that in the heavy particle limit it introduces all the (CP-even) dimension-6 operators that affect Higgs physics and are not severely constrained by electroweak precision physics.

In its simplest form, our model has three fixed and five free parameters. We take the free parameters to be the physics masses of the heavy scalar and top-quark partner, the mixing angles 


\begin{tabular}{|c|c|c|c|c|}
\hline $\mathrm{F}$ & $\varepsilon_{\gamma}$ & $\varepsilon_{g}$ & $\varepsilon_{B}$ & $\varepsilon_{W}$ \\
\hline \hline$\left(\begin{array}{c}T^{\prime} \\
B^{\prime}\end{array}\right)_{L+R}$ & $\frac{5}{18}$ & $-\frac{1}{6}$ & $\frac{1}{144}$ & $\frac{1}{16}$ \\
$Q_{L+R}$ & $\frac{1}{2} Q^{2}$ & $-\frac{1}{12}$ & $\frac{1}{8} Q^{2}$ & 0 \\
$\left(\begin{array}{c}N \\
E\end{array}\right)_{L+R}$ & $\frac{1}{6}$ & 0 & $\frac{1}{48}$ & $\frac{1}{48}$ \\
$L_{L+R}$ & $\frac{1}{16} Q^{2}$ & 0 & $\frac{1}{24}$ & 0 \\
\hline
\end{tabular}

Table 1: Possible vector-like fermion representations and their influence on the Higgs couplings to gauge fields. $Q^{2}$ represents the square of the fermion's electric charge and $\varepsilon_{V}$ is the derived coefficient of the $h V_{\mu v} V^{\mu v}$ operator.

$\theta$ in the scalar, and $\theta_{L}$ in the fermion sector, respectively, and the vacuum expectation value of the singlet field $v_{S}$, with the fixed parameters being the mass and vev of the SM-like Higgs as well as the top-quark mass:

$$
\begin{gathered}
m_{h_{2}}, \theta, v_{S}, m_{T} \text { and } \theta_{L} ; \\
m_{h_{1}}=125.0 \mathrm{GeV}, v_{H}=246 \mathrm{GeV}, m_{t}=173.2 \mathrm{GeV} .
\end{gathered}
$$

The free parameters of our simplified model are constrained by perturbative unitarity and electroweak precision data, as well as the Higgs cross sections and branching ratios.

\section{Higgs Couplings and Constraints}

We are primarily interested in the couplings of the light scalar $h_{1}$ (the $125 \mathrm{GeV}$ Higgs boson) to the Standard Model fermions of the third generation $(t, b, \tau)$ and the gauge bosons $\left(V=W^{ \pm}, Z, \gamma, g\right)$, parametrized in terms of the scale factors $\kappa \equiv g / g^{\mathrm{SM}}$ :

$$
\begin{aligned}
\mathscr{L}_{\mathrm{Higgs}} & =\kappa_{W} g_{h W W}^{\mathrm{SM}} h_{1} W^{+\mu} W_{\mu}^{-}+\kappa_{Z} g_{h Z Z}^{\mathrm{SM}} h_{1} Z^{\mu} Z_{\mu} \\
& -\kappa_{t} g_{h t t}^{\mathrm{SM}} h_{1} \bar{t} t-\kappa_{b} g_{h b b}^{\mathrm{SM}} h_{1} \bar{b} b-\kappa_{\tau} g_{h \tau \tau}^{\mathrm{SM}} h_{1} \bar{\tau} \tau \\
& +\kappa_{g} g_{h g g}^{\mathrm{SM}} h_{1} G^{\mu v} G_{\mu v}+\kappa_{\gamma} g_{h \gamma \gamma}^{\mathrm{SM}} h_{1} A^{\mu v} A_{\mu v} .
\end{aligned}
$$

In our model, the tree-level couplings to $V=W^{ \pm}, Z$ and to the fermions other than the topquark, are only modified due to the mixing of the Higgs fields $h$ and $s, h_{1}=h \cos \theta-s \sin \theta$, as the singlet field $s$ does not couple directly to the SM fields. Thus we have

$$
\kappa_{W}=\kappa_{Z}=\kappa_{b}=\kappa_{\tau}=\cos \theta .
$$

The couplings to the top-quark are modified through both the mixing of the scalar fields and the mixing in the top-quark sector. For the coupling of the light scalar, $h_{1}$, with the light top-quark, $t$, we find

$$
\kappa_{t}=\cos ^{2} \theta_{L} \cos \theta-\sin ^{2} \theta_{L} \sin \theta \frac{v_{H}}{v_{S}} .
$$


The loop-induced couplings to the gluon and the photon differ from those of the Standard Model because of the modified Higgs $W$ and top couplings as well as the additional contribution of the heavy-top loop. In the limit $m_{h_{1}} \ll m_{t}, m_{T}$ we have

$$
\kappa_{g} \approx \cos \theta-\sin \theta \frac{v_{H}}{v_{S}} .
$$

For the other couplings we refer the reader to Ref. [4].

Since the Higgs couplings to the $W$ and $Z$ bosons are the most accurately measured to date, they will provide the strongest constraints on the mixing angle $\theta$ (especially since the photonic couplings are affected by the presence of the top partners $T$ ). We can also derive a limit on $\theta_{L}$, and a weak bound on $m_{T}$, from $\kappa_{g}$ and $\kappa_{\gamma}$, although the dependence on these parameters in these loop-induced modes is mild. The 5th parameter of the model, the mass of the heavy scalar $m_{h_{2}}$, does not enter the $125 \mathrm{GeV}$ Higgs couplings to Standard Model fermions and gauge bosons and we will find it to be less constrained by Higgs searches.

Fig. 2 shows the result of a global fit of this simplified model to the Higgs and electroweak precision data [4]. We see that the distribution in $m_{h_{2}}$ is relatively flat for $m_{h_{2}}$ above $\sim 200 \mathrm{GeV}$, since once $\sin \theta$ is small the $h_{2}$ production cross-sections are suppressed, evading the LHC searches and limits. In turn, $\sin \theta$ is forced to be close to 0 (i.e., towards the SM limit) due to the good agreement between the experimentally measured Higgs properties and those predicted in the SM. Both the ratios $v_{H} / v_{S}$ and $m_{t} / m_{T}$ are already driven towards the decoupling limit by current measurements of the Higgs properties (as well as the $S$ and $T$ parameters), with $68 \%$ confidence intervals restricting $v_{H} / v_{S} \leq 0.85$ and $m_{t} / m_{T} \leq 0.42$.

\section{Collider Phenomenology of the Scalar Sector}

In this section, we examine the phenomenology of the $h_{1,2}$ scalar bosons at the LHC. We first explore effects of $h$-S mixing on $h_{1}$ production rates to determine the degree of difference from Standard Model expectations and then study $h_{2}$ production and decay.

The Higgs plus jet cross section, $p p \rightarrow h_{1}+$ jet, is shown in Fig. 3 (left) as a function of a cut on the $p_{\perp}$ of the Higgs. We have set $\sin \theta_{L}=\sin \theta=0.15$ (which is at the outer envelope of the allowed parameter space from the fit, and thus provides an upper bound on how large deviations from the SM can be), and consider two choices for the mass of the vector-like $T$ quark, $m_{T}=500 \mathrm{GeV}$ and $m_{T}=1 \mathrm{TeV}$, respectively. The lower of these masses for $m_{T}$ is naively ruled out by current LHC searches, however in models such as ours which allow for dilution of the standard $T$ branching ratio the limits may be lower. In the case our choice of $m_{T}=500 \mathrm{GeV}$, similar to the choices for $\sin \theta$ and $\sin \theta_{L}$ allows us to estimate the maximum size of the deviations away from SM behaviour.

While the emission of a hard jet allows for the exploration of the structure of the heavy fermion loop in principle, we see that the deviations from the SM prediction, which ranges from $1.63 \mathrm{pb}$ for $p_{\perp}>100 \mathrm{GeV}$ to $4.5 \mathrm{fb}$ at $p_{\perp}>500 \mathrm{GeV}$, turn out to be numerically small in our model, given the allowed range of masses and mixings. It will thus be challenging to constrain the properties of the vector-like $T$ quark from a measurement of the Higgs+jet cross section. Another way to probe new physics in the Higgs coupling to gluons is through double Higgs production. We show the corresponding cross section, $p p \rightarrow h_{1} h_{1}$ in Fig. 3 (right). Here, we have again set $\sin \theta_{L}=\sin \theta=$ 

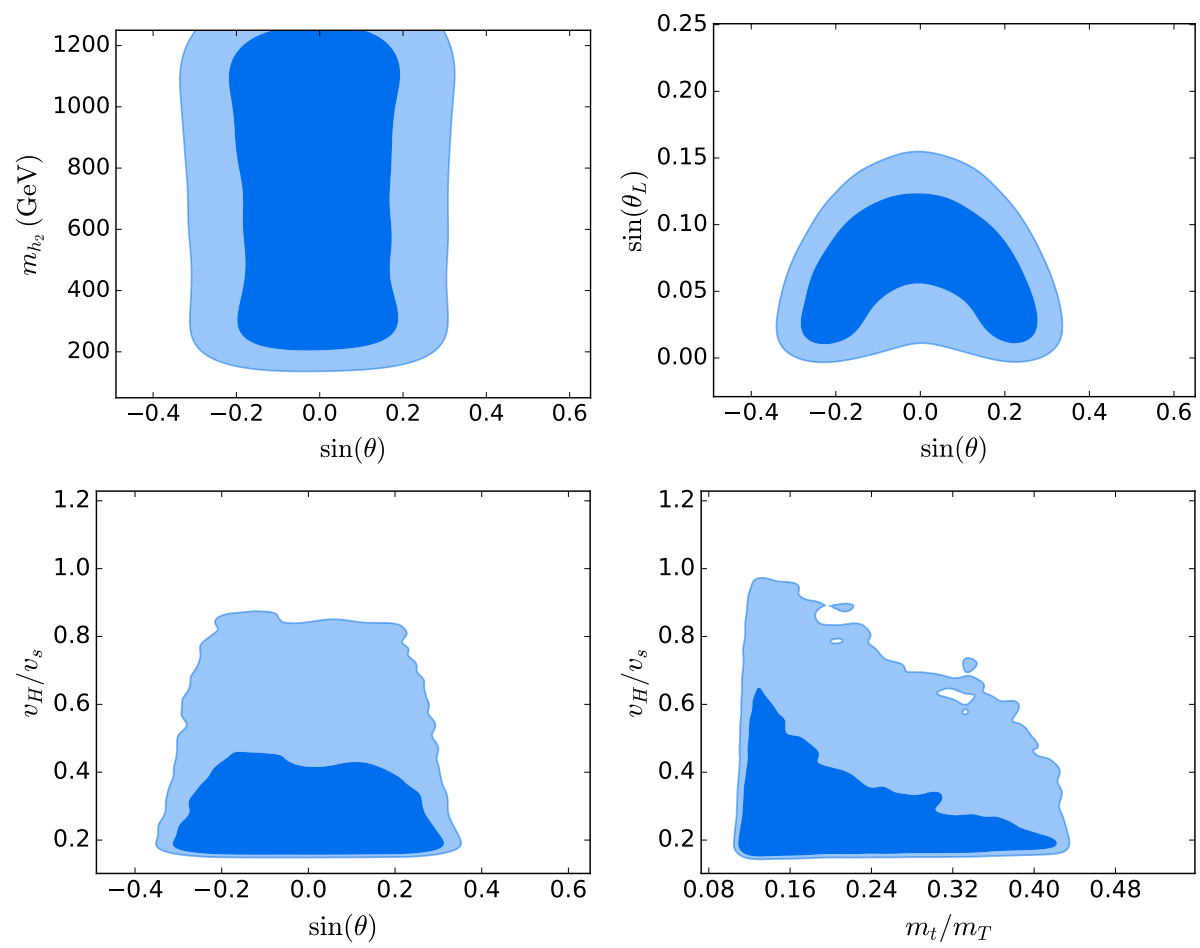

Figure 2: 68 and 95\% Bayesian confidence limit contours (light and dark blue respectively) obtained from the fit to Higgs and precision electroweak data in the (clockwise from top-left) $\sin \theta-m_{h 2}, \sin \theta-\sin \theta_{L}, \sin \theta$ $v_{H} / v_{S}$ and $m_{t} / m_{T}-v_{H} / v_{S}$ parameter planes.

0.15 , and explore the cross section as a function of the mass of the vector-like $T$ quark, $m_{T}$, the singlet vacuum expectation value, $v_{S}$, and the mass of the heavy scalar, $m_{h_{2}}$. We find potentially sizable deviations from the $\mathrm{SM}$ cross section, $\sigma_{\mathrm{SM}}=14.6 \mathrm{fb}$, resulting from the resonant production $p p \rightarrow h_{2} \rightarrow h_{1} h_{1}$. The size of the cross section depends not only on the mass of the heavy scalar, but also on the vacuum expectation value $v_{S}$ and the mass of the vector-like quark, which leads to a second threshold at $m_{h_{2}} \sim 2 m_{T}$. The observation of double Higgs production would thus be important to probe new physics in the loop-level coupling of the Higgs to gluons.

\section{5. $T$ Production and Decay at the LHC}

As is the case for any new color-triplet fermion, $T \bar{T}$ production proceeds at leading order in QCD via both $q \bar{q}$ and $g g$ annihilation, analogous to $t \bar{t}$ production in the SM. We show an example Feynman diagram for the $g g \rightarrow T \bar{T}$ process in Fig. 4 left in the upper-left hand corner. This production rate is quite large even for $T$ masses of order $\sim 1 \mathrm{TeV}$ as can be seen in the righthand panel of Fig. 4 which shows the cross-section at $13 \mathrm{TeV}$ as a function of the $T$-quark mass. Resonant $T \bar{T}$ production can also occur through gluon fusion $g g \rightarrow h_{2} \rightarrow T \bar{T}$ when $2 m_{T} \leq m_{h_{2}}$. Given the current limits on $m_{T}$, this is unlikely to be relevant at the LHC, although it may be observable at a future higher-energy hadron collider. 

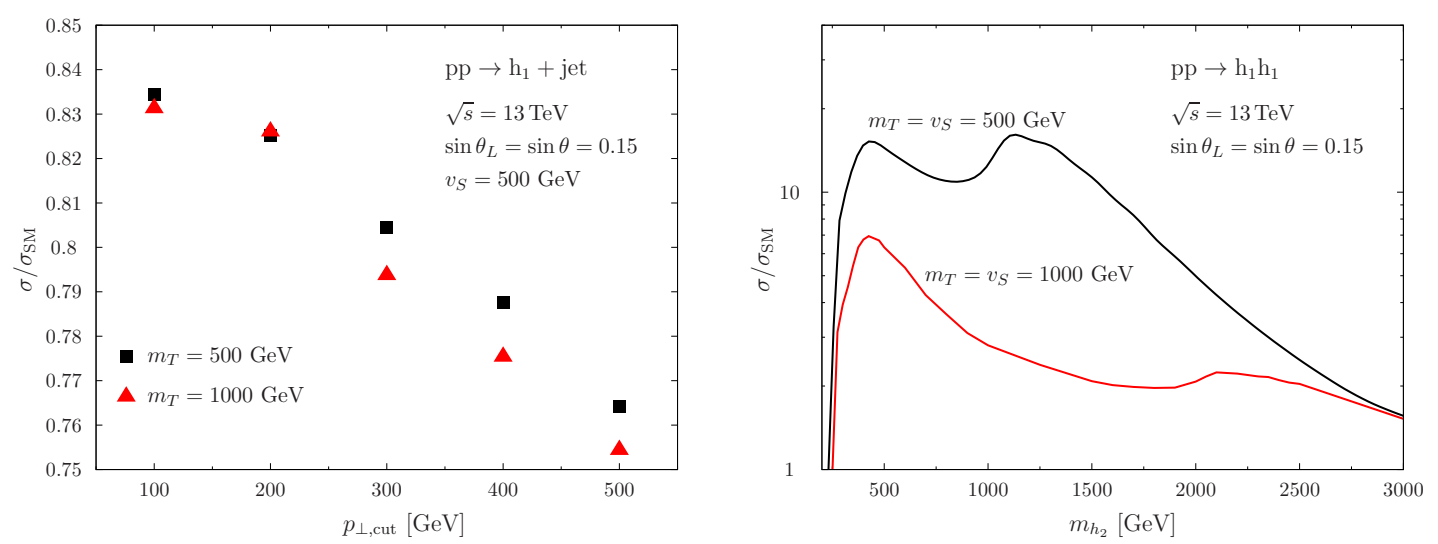

Figure 3: (Left) The $125 \mathrm{GeV}$ Higgs plus jet cross section, $p p \rightarrow h_{1}+$ jet, as a function of a cut on the $p_{\perp}$ of the Higgs for $\sqrt{s}=13 \mathrm{TeV}$. Shown is the ratio of the simplified Higgs model and the SM prediction, both at leading order, for different values of $m_{T}$. (Right) Higgs pair production, $p p \rightarrow h_{1} h_{1}$, at $13 \mathrm{TeV}$, relative to the SM prediction, for two different values of $m_{T}$ and $v_{S}$. In both panels we have set $\sin \theta_{L}=\sin \theta=0.15$.
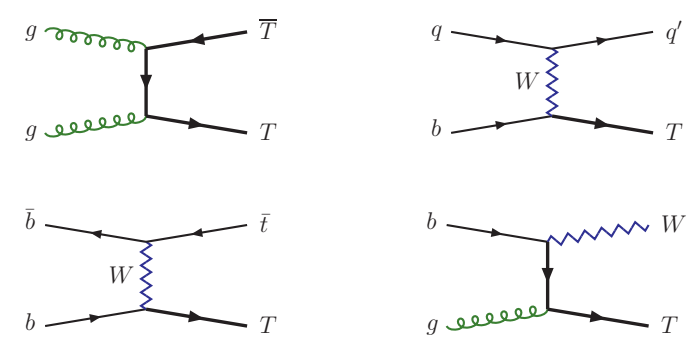

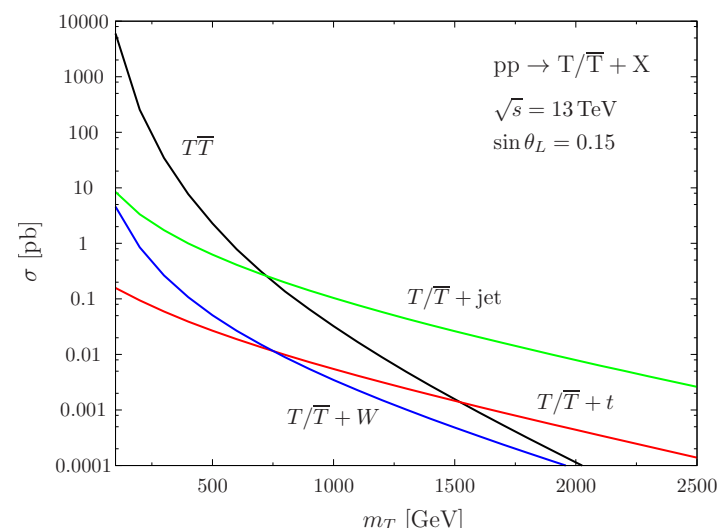

Figure 4: (Left) Exemplary Feynman diagrams for $T \bar{T}$ (top-left) and single $T$ production at the LHC. (Right) The $g g, q \bar{q} \rightarrow T \bar{T}$ production cross section at $13 \mathrm{TeV}$ (black) and the corresponding single $T$ production by several mechanisms: $q b \rightarrow q^{\prime} T$ (i.e., $T+$ jet in green), $g b \rightarrow W T$ (i.e., $T+W$ in blue) and $q \bar{q}, b \bar{b} \rightarrow T t$ (i.e., $T+t$ in red). We have taken $s_{L}=0.15$.

Due to the potentially significant $t-T$ mixing within the present scenario, somewhat more interesting single- $T$ production processes can also occur; examples of these production mechanisms are depicted in the left-hand panel of Fig. 4. It is clear that single $T$-quark production can occur via several channels, in analogy to single top production in the SM. This can occur in a variety of ways, for example via $t$-channel $W$-exchange, $q b \rightarrow q^{\prime} T$, which corresponds to single top production in the SM except that now the $T$ is more massive than the $t$ and the coupling, as discussed above, is suppressed by an additional factor of $s_{L}$. Similarly, $W+T$ associated production, $g b \rightarrow W T$, can occur via $s$-channel $b$-exchange as well as $t$-channel $T$-exchange which is again analogous to single $t$ production in the SM except for the larger $T$ mass and the amplitude-level suppression by a factor of $s_{L}$. Taking $s_{L}=0.15$ as a large, but allowed, value of the mixing given our above fit, Fig. 4 shows that these cross sections can be quite significant, particularly for large $T$ masses. We find that for 
$m_{T} \gtrsim 750 \mathrm{GeV}$ the $T+$ jet single production rate is larger than that for pair production from QCD due to the significant phase-space suppression. For other values of $s_{L}$, the cross sections for these processes can be easily obtained from the figure by rescaling by a factor of $\left(s_{L} / 0.15\right)^{2}$. Finally, the single $T$ production process $q \bar{q}, b \bar{b} \rightarrow \bar{t} T+$ h.c. can also now occur via $t$-channel $W$-exchange as well as $s$-channel flavor-changing $Z$-exchange which leads to the smallest rate shown in the figure. Recall that this flavor-changing coupling arises from the fact that $t_{L}$ and $T_{L}$ have different values of the third component of weak isospin, $T_{3 L}$. In this case the cross section scales as $\left(s_{L} c_{L}\right)^{2}$.

Of course $t \bar{T}+$ h.c. production can also occur from both $g g$ and $q \bar{q}$ initial states via QCD through a loop-induced flavor-changing $(t \bar{T}+T \bar{t}) g$ coupling. Such an interaction vertex will be not only loop-suppressed but will also involve the familiar factors of the $t-T$ mixing angle, $s_{L}$, leading to an even further suppression, and we do not consider it further in this work.

$T-t$ mixing allows for the decay of the $T$-quark and without which $T$ would be stable. Within the present framework, any discussion of $T$ decays (and the collider searches for $T$ ) necessarily involves the role of the additional mostly-singlet Higgs field, $h_{2}$. The tree-level decay modes of $T$ (if they are kinematically allowed) are $T \rightarrow W b, Z t, h_{1} t$ and $h_{2} t$, all of whose corresponding partial widths are proportional to the factor $s_{L}^{2}$. The presence of this 'exotic' $h_{2} t$ decay mode, which is absent in almost all discussions of vector-like heavy quark decays, can have a strong potential impact on the searches for $T$-quarks that have been performed so far at the LHC. At present, existing searches only consider the case where the SM is solely augmented by a heavy $T$-quark with no additional $h_{2}$ field being present. Thus these analyses all make the common assumption that the sum of the branching fractions for the $W b, Z t$ and $h_{1} t$ decay modes must sum to unit. In addition, as these standard searches rely on reconstructing the $T$ mass from the SM decay products, they have minimal sensitivity to the $T \rightarrow h_{2} t$ decay channel (the exception to this would be if $m_{h_{1}} \sim m_{h_{2}}$ such that the acceptances would be similar). However, as we will see below, there can be a significant region of the model parameter space where the $h_{2} t$ decay mode is kinematically allowed and obtains a respectable coupling strength relative to that for $h_{1} t$. Clearly the influence of this mode on the lower bound obtained on the $T$ mass will depend upon how $h_{2}$ itself decays. We might expect that if the $h_{2}$ decays in a manner broadly similar to the SM-like $h_{1}$ (e.g. into $W W, Z Z$ or $b \bar{b}$ ) the effects will be minimal except that the final state kinematics can be significantly different depending upon both the $T$ and $h_{2}$ masses. Of course if $h_{2}$ is sufficiently heavy, the branching ratio for the decay $h_{2} \rightarrow t \bar{t}$ can be significant so that the decay path $T \rightarrow t t \bar{t}$ via virtual $h_{2}$ exchange opens up, this has also not been examined in $T$-quark searches. Clearly a detailed analysis of how the existence of a non-negligible branching fraction for $T$ into the $h_{2} t$ mode would affect the searches for $T$-quarks at the LHC remains an open question that needs to be performed in detail, but we might expect that if this branching fraction is sufficiently small the 'standard' limits discussed above will approximately apply.

To provide some overall understanding of the interplay between the masses of $h_{2}$ and $T$ and the corresponding decay physics, the left-hand panel of Fig. 5 gives a semi-quantitative feel for the regions in this space where the various decay modes may occur. The strengths of the various couplings are determined by the three remaining model parameters $s_{\theta}, s_{L}$ and the ratio of vev's $r=v_{H} / v_{S}$ and are given in [4]. An important example is provided by the ratio of the $T \rightarrow h_{2} t$ and 

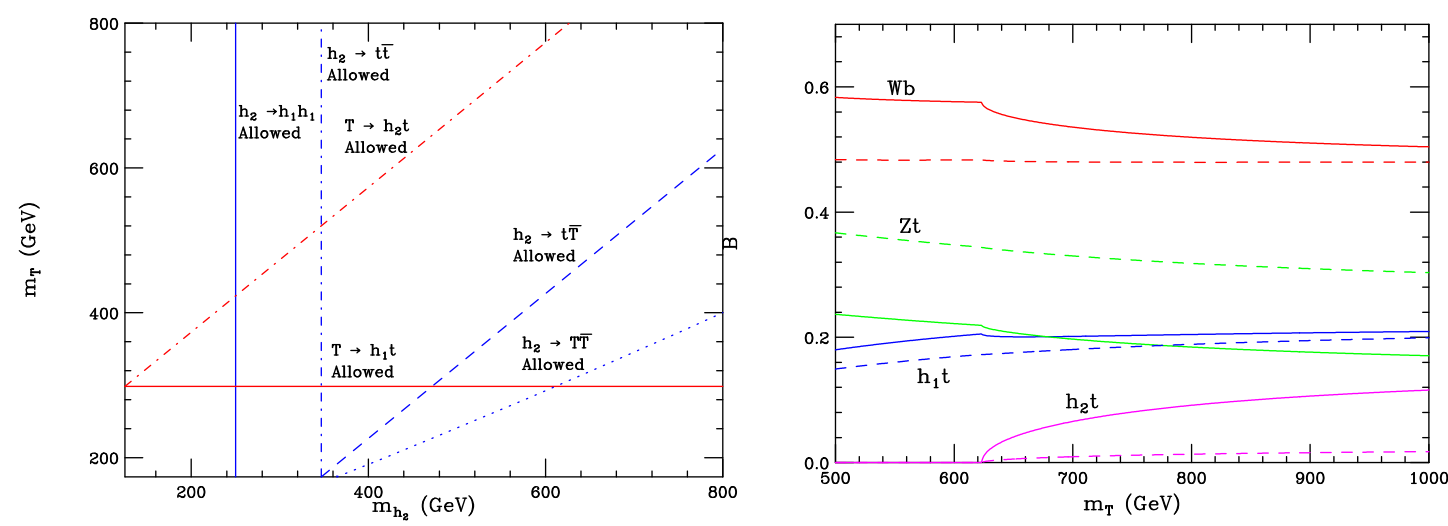

Figure 5: (Left) Kinematic regions for the various decays of $h_{2}$ and $T$ in the $m_{h_{2}}-m_{T}$ plane. To the right of the blue solid (dash-dotted, dashed, dotted) lines the decay $h_{2} \rightarrow h_{1} h_{1}(t \bar{t}, t \bar{T}+$ h.c.,$T \bar{T})$ are kinematically allowed. In the region above the red solid (dash-dotted) line the decay $T \rightarrow h_{1} t\left(h_{2} t\right)$ is kinematically allowed. (Right) Sample $T$ decay branching fractions as a function of $m_{T}$ assuming $m_{h_{2}}=300 \mathrm{GeV}, s_{L}=0.15$, $v_{S}=500 \mathrm{GeV}$ and $s_{\theta}=-0.15(0.15)$ corresponding to the dashed (solid) curves. The branching fractions for the $\mathrm{Wb}\left(\mathrm{Zt}, h_{1} t, h_{2} t\right)$ modes are shown as the red (blue, green, magenta) curves.

$T \rightarrow h_{1} t$ partial widths, $R$, which apart from phase space factors is given by

$$
R=\frac{\Gamma\left(T \rightarrow h_{2} t\right)}{\Gamma\left(T \rightarrow h_{1} t\right)} \sim\left[\frac{s_{\theta}-r c_{\theta}}{c_{\theta}+r s_{\theta}}\right]^{2},
$$

where $s_{\theta}=\sin \theta$ and $c_{\theta}=\cos \theta$. Clearly, the parameter space region with somewhat larger $r$ values and with $s_{\theta}<0$ can lead to a significant result for this ratio, thus providing a good example of a situation where the sign of $s_{\theta}$ is important. In Fig. 5 above, we see that there is a region where $R>1$ is obtained.

The right-hand panel of Fig. 5 compares the branching fractions for the various $T$-quark decay modes for two parameter choices which differ only in the choice of sign for $s_{\theta}$. There can be some significant differences in these two cases: apart from the obviously much larger value for the branching fraction of the $h_{1} t$ mode when $s_{\theta}>0$ we see that the branching fraction for $h_{2} t$ is larger in the $s_{\theta}<0$ case at the expense of that for $h_{1} t$ at larger values of $m_{T}$. On the other hand, we also see that the branching fractions for the $W b, Z t$ decay modes are relatively unaffected by the change of sign of $s_{\theta}$. In general, since $\kappa_{g} \simeq c_{\theta}-s_{\theta} r$, much of the parameter space where the above ratio of decay widths is large is restricted by LHC data at some level since it simultaneously produces a too large value for $\kappa_{g}$.

\section{Conclusion}

We have formulated a set of simplified models to characterize the interactions of physics beyond the Standard Model with the $125 \mathrm{GeV}$ Higgs boson. We selected one such model, where the Standard Model is extended by a gauge singlet scalar and vector-like fermions which mix with 
the SM top-quarks, and studied its phenomenology in detail. In particular, we examined the complementarity between indirect searches for new physics in precise determinations of the $125 \mathrm{GeV}$ Higgs couplings and distributions with direct searches for new particles. We constrained the model parameters by performing a global fit using the ATLAS and CMS combined data on the Higgs couplings and searches for heavy new scalars. We argued that although our model allows for the possibility of exotic top partner phenomenology, it is robustly constrained by top partner searches from LHC Run 1. Nonetheless, the top-partner decay into a SM top-quark and new heavy scalar, $T \rightarrow t h_{2}$, would lead to novel signatures and deserves to be better explored with Run 2 searches in mind. We find that given current direct search constraints, the effects of the top partners in Higgs loops are mostly likely too small to observe, in line with general expectations in weakly coupled models. As the LHC moves further into the precision era, simplified models for Higgs physics will serve as a test-bed for expectations of possible BSM signals in the Higgs sector and direct searches for new particles.

\section{Acknowledgement}

The research of MD was performed in part at the Munich Institute for Astro- and Particle Physics (MIAPP), part of the DFG cluster of excellence "Origin and Structure of the Universe". MK would like to thank SLAC and the SLAC Theory group for hospitality and support. The work of MK is supported by the German Research Foundation DFG through the research unit 2239 "New physics at the LHC". The work of JLH and TGR was supported by the U.S. Department of Energy, Contract DE-AC02-76SF00515.

\section{References}

[1] G. Aad et al. [ATLAS and CMS Collaborations], Measurements of the Higgs boson production and decay rates and constraints on its couplings from a combined ATLAS and CMS analysis of the LHC pp collision data at $\sqrt{s}=7$ and $8 \mathrm{TeV}$, JHEP 1608 (2016) 045, [arXiv:1606.02266 [hep-ex]].

[2] J. Alwall, P. Schuster and N. Toro, Simplified Models for a First Characterization of New Physics at the LHC, Phys. Rev. D 79 (2009) 075020, [arXiv:0810.3921 [hep-ph]].

[3] D. Alves et al. [LHC New Physics Working Group Collaboration], Simplified Models for LHC New Physics Searches, J. Phys. G 39 (2012) 105005, [arXiv:1105.2838 [hep-ph]].

[4] M. J. Dolan, J. L. Hewett, M. Krämer and T. G. Rizzo, Simplified Models for Higgs Physics: Singlet Scalar and Vector-like Quark Phenomenology, JHEP 1607 (2016) 039, [arXiv:1601.07208 [hep-ph]]. 